\title{
Artificial Bee Colony algorithm for Traveling Salesman Problem
}

\author{
Hongwei Jiang ${ }^{1, a}$ \\ ${ }^{1}$ School of Information Management, Beijing Information Science and Technology University, \\ Beijing, 100192, China \\ aemail: jianghongwei@bistu.edu.cn
}

Keywords: Traveling Salesman Problem; Artificial Bee Colony; Combinatorial Optimization

\begin{abstract}
Travelling salesman problem (TSP) is a fundamental combinatorial optimization model studied in the operations research community for nearly half a century. It belongs to the class of NP-Complete problems. It has been proved that evolutionary algorithms are effective and efficient, with respect to the traditional methods for solving NP-Complete problems like TSP, with avoidance trapping in local minima areas. Artificial Bee Colony (ABC) is a new swarm-based optimization algorithm, which inspired by the foraging behavior of honey bees. This paper uses artificial bee colony algorithm to solve traveling salesman problems, gives the specific solutions of artificial bee colony algorithm for solving traveling salesman problem, and makes simulation experiment for the problem. The results show that the algorithm can efficiently and quickly find optimal or sub-optimal solutions.
\end{abstract}

\section{Introduction}

The travelling salesman problem (TSP) states that for one salesman who wants to visit cities and given distances between them. Travelling salesman has to visit all of them, but he does not to travel very much. Task is to find a sequence of cities to minimize travelled distance [1]. TSP is an NP-hard problem in combinatorial optimization studied in operations research. TSP has several applications, such as logistics, transportation.

In recent years many approaches have been developed to solve TSP that can be divided to exact [2] and approximate approaches [3]. Exact methods can only be used for very small size instances, and for real-world problems, researchers have to apply approximate methods that find near-optimal solutions in a reasonable time rather than an exact method that guarantees to find the optimal solution in an exponential time.

In this paper we solve the travelling salesman problem using the real version of ABC algorithm. The rest of paper is organized as follows: In section 2, the ABC algorithm has reviewed and presents the methodology of ABC algorithm. Solution methodology and experimental setup proposed for TSP are given in section 3. The experimental results are shown in section 4, and consequently, a conclusion is described in section 5.

\section{Artificial Bee Colony Algorithm}

The Artificial Bee Colonies (ABC) is a novel optimization algorithm that comes under Swarm Intelligence. $A B C$ algorithm is inspired by social behavior of natural bees. The Artificial Bee Colony, introduced by Dervis Karaboga in 2005 (Karaboga, 2005; Karaboga \& Basturk, 2007) [4][5].The natural bees are very good in searching for some food source. Whenever any bee finds the food, it signals the other bees by its dance. This signals the other bees regarding the quantity and the location of the food source. This helps in directing the other bees towards good sources of food in their search for food. These bees are able to attract a large number of bees and carry forward to search the food area.

In ABC algorithm the bees are divided into three groups: employed bees, onlooker bees and scout bees. Employed bees visited the food source and gather information about food source location and the quality. Employed bees have memory, so they know the places they have visited 
before and the quality of food there. Employed bees performs the local search and try to exploit the neighboring locations of the food source and search the best places of foods in the surrounding areas of the present value. Onlooker bees are bees that are waiting on the dance area to decide which food source is better. This decision is made on the basis of information provided by employed bees. Onlooker bees perform the global search for discovering the global optimum. Scout bees do a random search for the food. Scout bees discovers the new area which are uncovered by the employed bees, these bees are completely random in nature and their operation of search. Scout bees avoid the search process to get trapped in local minima. In ABC algorithm, first half of the colony consists of employed bees and the second half constitutes by onlooker bees. The number of employed bees is equal to the number of food sources around the hive. The employed bee whose food source is exhausted becomes the scout bees. In ABC algorithm each food source position represents a candidate solution of optimization problem. In optimization problem each solution is associated with the fitness value on the basis of fitness value it is decided that which solution is better. So the nectar amount of a food source corresponds to the fitness value of the associated solution in ABC algorithm. The number of employed bees or the onlooker bees is equal to the number of solutions in the population.

The ABC algorithm generates a random solution or initial population of size $\mathrm{N}$, where $\mathrm{N}$ denotes the size of population or total number of food source.

Food source $X_{i}(i=1, \ldots, N)$ are vectors of $D$ dimension, which corresponding to one employed bee. $\mathrm{F}(\mathrm{Xi})$ is the fitness value of the solution $\mathrm{Xi}$ or the nectar amount of food source. The iteration times are constrained to "Limit" for each source, and max iteration is constrained to MAX $_{\text {pop }}$ for whole population.

Step one: generate $\mathrm{N}$ feasible solution randomly.

$$
\begin{array}{ll}
\text { fo } & \text { For } \mathrm{i}=1 \text { to } \mathrm{N} \\
& \mathrm{X}_{\mathrm{i}}^{\mathrm{j}}=\mathrm{X}_{\min }^{\mathrm{j}}+\operatorname{rand}(0,1)\left(\mathrm{X}_{\max }^{\mathrm{j}}-\mathrm{X}_{\min }^{\mathrm{j}}\right) \quad(\mathrm{j} \text { is component of } \mathrm{D} \text { dimension vector })
\end{array}
$$

Step two: employed bee search good solution in food source neighbor, and calculate the fitness value, if new source fitness is higher than old one, replace it. Search new source formula as follows: For $\mathrm{i}=1$ to $\mathrm{N}$

do

$$
V_{i}^{j}=X_{i}^{j}+\varphi_{i}^{j}\left(X_{i}^{j}-X_{k}^{j}\right)
$$

(new source fitness is $F\left(V_{i}\right)$, if $F\left(X_{i}\right)$ lower than $F\left(V_{i}\right)$, make $V_{i}$ replace $X_{i}$, otherwise unchanged.)

Step three: for follow bees, according to the probability of positive correlation to employed bee fitness value, choosing one employed bee to neighborhood search. The probability of food source i is chosen is:

$$
P i=\frac{F(X i)}{\sum_{i}^{n} F(X i)}
$$

(The ways follow bees search and select the food source is similar to step 2)

Step four: for all food source, if search times reach a certain limit but still do not find better solution around some food source, give up the source and generate new source:

For $\mathrm{i}=1$ to $\mathrm{N}$

do limit $=0 ;\}$

$$
\begin{aligned}
& \{\text { limit }=0 ; \mathrm{m}=0 ; \\
& \text { If } \mathrm{F}\left(\mathrm{X}_{\mathrm{m}}\right)=\mathrm{F}\left(\mathrm{X}_{\mathrm{m}-1}\right) \text { then } \mathrm{m}=\mathrm{m}+1 \text {; } \\
& \text { If } \mathrm{m}=\text { Limit then }
\end{aligned}
$$

\{give up the food source, and generate a new source according to formula(1.1); \}

Step five: Judge whether algorithm meets the end conditions, or get maximum iteration limit $\mathrm{MAX}_{\mathrm{pop}}$. If it is, output the optimal solution, otherwise the algorithm into the next iteration. 


\section{ABC approach to TSP}

TSP can be represented as a graph $G=(V, E)$, where $V=\{1,2, \ldots, M\}$ is a set of nodes, and $E$ $=\{(i, j) \mid i, j \in V\}$ is the set of all connection edges between them. Each node represents a city, and each edge means the possible path between two related cities. The distance $d_{i j}$ is associated with edge (i, j) and represents the Euclidean distance from city $i$ to city $j$. The heuristic information is calculated before performing the main algorithm. So, the distances of all edges were calculated and saved. Iteratively, after construction the solutions, the qualities of the gathered solutions are evaluated by the fitness value, which is calculated by sum of the Euclidean distances of the consecutive edges within the tour. To solve TSP using ABC algorithm, at the first step, an initial population (food source positions) is generated via random way considering diversity of population.

For every algorithm there are some control parameters which are used for its efficient working. Hence, there are some control parameters for artificial bee colony algorithm also. We did an extensive literature survey and carried out our own experiments for determining the values of these control parameters. From this we found that the values which we have taken in this experiment are standard values and they are also suitable for this experiment. The first control Parameter is Maximum cycle number: Maximum number of cycles (MCN) equals to the maximum number of generation, we have taken the result for $3000 \mathrm{MCN}$ value. The next parameter in our experiment is maximum number of population and we have taken its value to be 50 . Another control parameter is number of runs and we have taken its value in our experiment as 40 . It must be noted that each run contains maximum cycle number, which is 3000 in our experiment. The fourth control parameter is Dimension and it depends upon the number of city.

\section{Computational Results}

In this section we have tested our approach on the travelling salesman problem with 15 cities. Figure 1 is the path diagram without being optimized and figure 2 is the path diagram with being optimized. Figure 3 is the convergent iteration curve with ABC algorithm. It can be seen that the application of artificial bee colony algorithm, the path has been significantly optimized. With the completion of the iteration, the path length can quickly converge to the shortest path length. We can also use our proposed algorithm ABC to solve similar optimization problems.

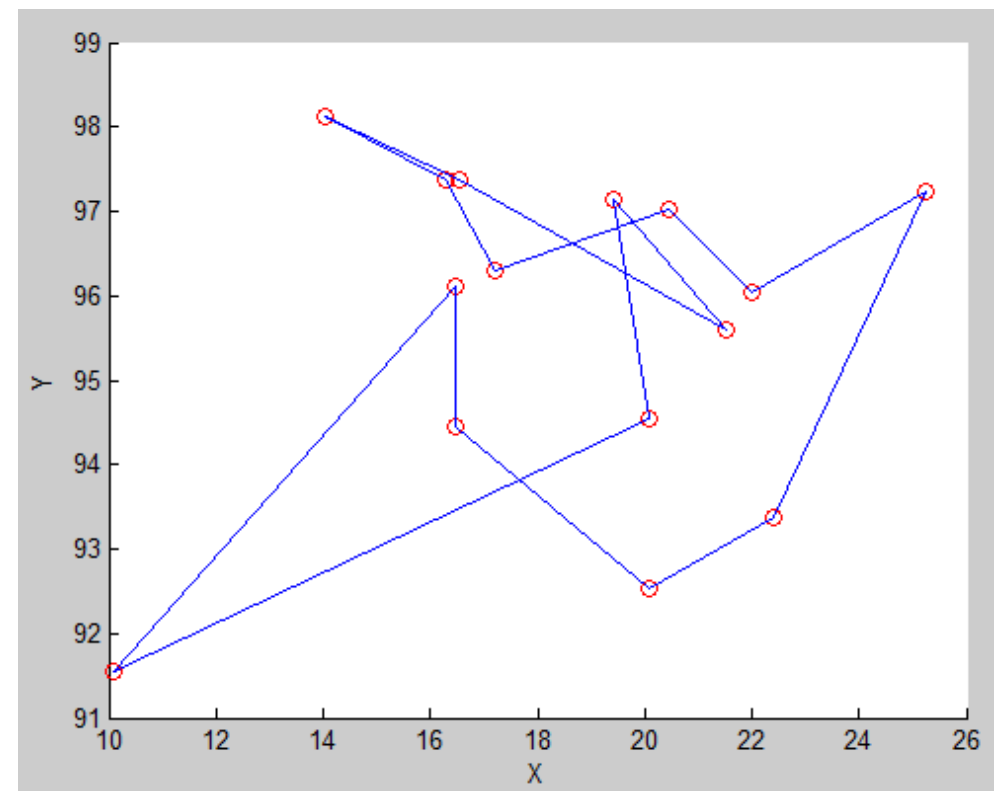

Fig.1. Path diagram for TSP without being optimized 


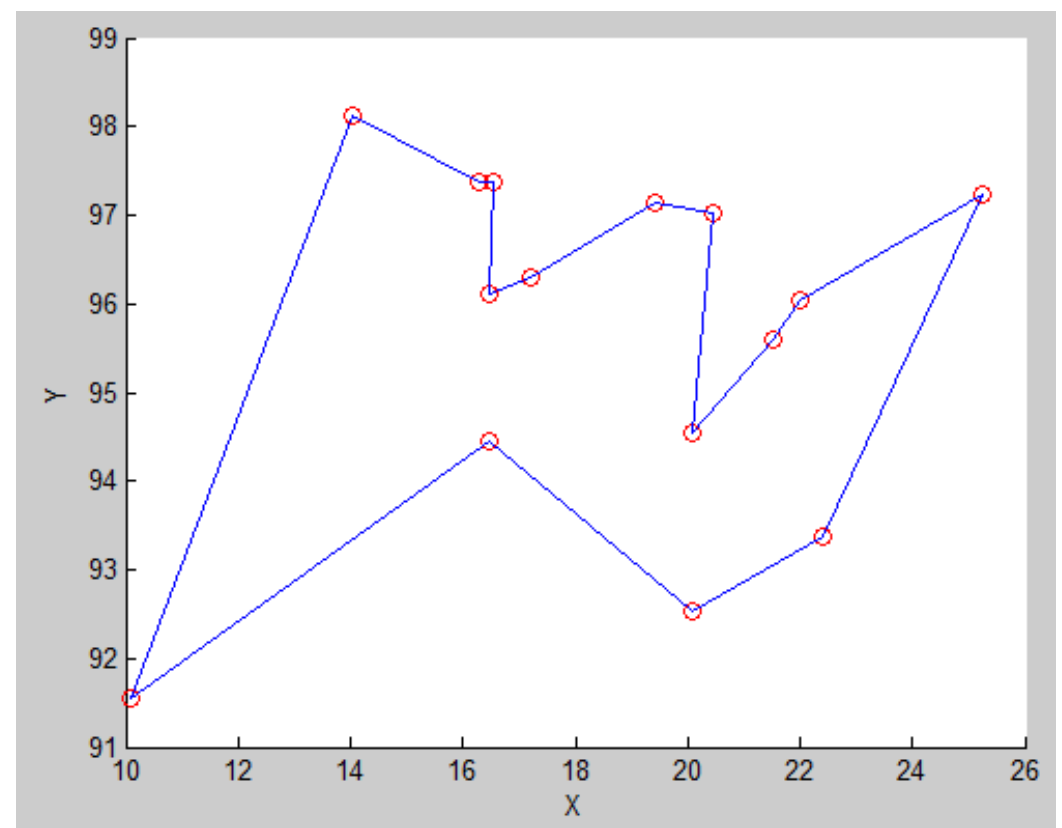

Fig.2. Path diagram for TSP with being optimized

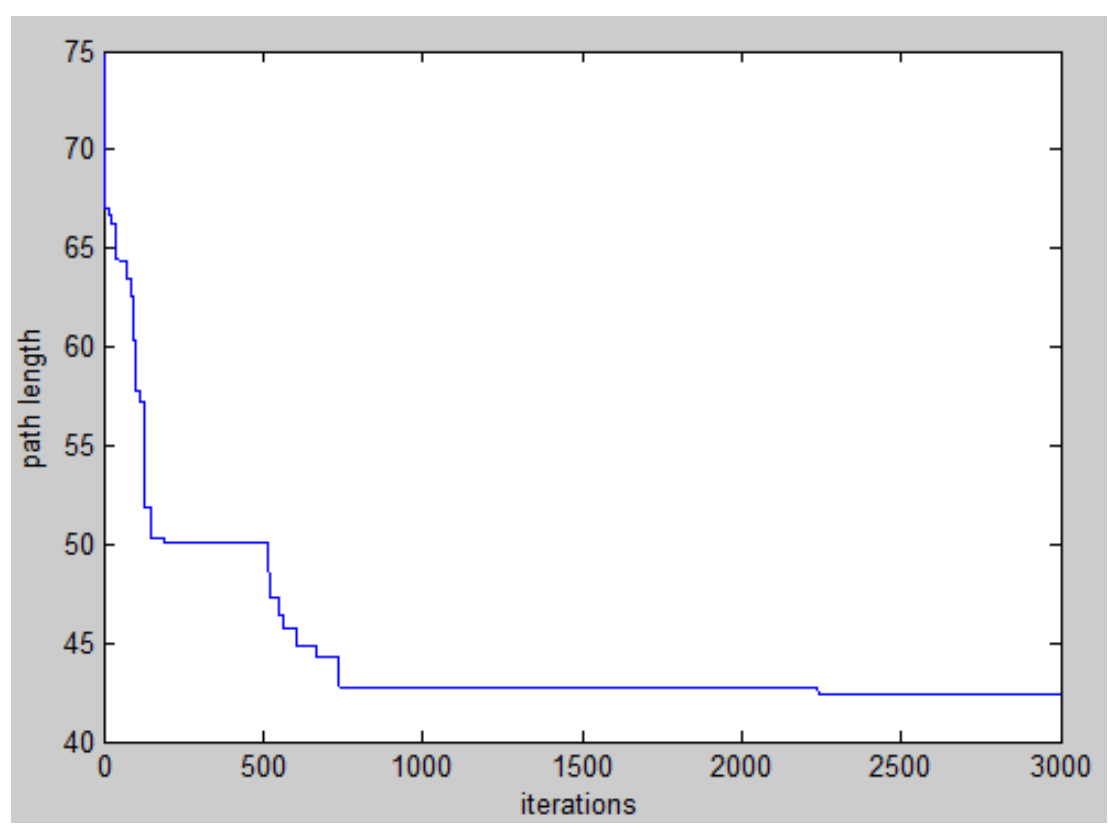

Fig.3. Convergent iteration curve with ABC algorithm

\section{Conclusion}

In this paper, $\mathrm{ABC}$ algorithm was introduced for TSP aims to efficiently reduce the complexity of evolutionary algorithms. ABC algorithm has a strong search capability in the graph space and can effectively find an optimal path. Experimental results show that this approach considers both running time and solution quality as well. As a future work, the algorithm will be hybridized with other algorithm to find better results.

\section{Acknowledgement}

The research work was supported by Foundation for the Importation and Development of High-Caliber Talents Project of Beijing Municipal Institutions (Grant NO. CIT\&TCD201304118). 


\section{References}

[1] G. Reineit. TSPLIB-A traveling salesman problem library[J]. ORSA Journal on Computing, 1991,3(4) 376-384.

[2] T. Bektas. The multiple traveling salesman problem: an overview of formulations and solution procedures[J]. Omega, 2006 34(3) 209-219.

[3] A. Ugur and D. Aydin. An interactive simulation and analysis software for solving TSP using ant colony optimization algorithm[J]. Advances in Engineering Software, 2009 40(2) 341-349.

[4] D. Karaboga. An idea based on honey bee swarm for numerical optimization[R]. Erciyes University, Engineering Faculty, Computer Engineering Department, Turkey, Technical Report-TR06, 2005.

[5] D. Karaboga, B. Akay. A Survey: Algorithms Simulating Bee Swarm Intelligence[J]. Artificial Intelligence Review, 2009 31(1) 68-85. 